\title{
Evolution of a Water Pendant Droplet: Effect of Temperature and Relative Humidity
}

\author{
Etienne Portuguez¹, Arnaud Alzina1, Philippe Michaud1', Maksoud Oudjedi², Agnès Smith ${ }^{*}$ \\ ${ }^{1}$ Université de Limoges, SPCTS, CNRS, ENSCI, Limoges, France \\ ${ }^{2}$ Université de Limoges, ENSCI, Limoges, France \\ Email:*agnes.smith@unilim.fr
}

How to cite this paper: Portuguez, E. Alzina, A., Michaud, P., Oudjedi, M. and Smith, A. (2017) Evolution of a Water Pendant Droplet: Effect of Temperature and Relative Humidity. Natural Science, 9, 1-20. http://dx.doi.org/10.4236/ns.2017.91001

Received: December 1, 2016

Accepted: January 14, 2017

Published: January 17, 2017

Copyright ( $\odot 2017$ by authors and Scientific Research Publishing Inc. This work is licensed under the Creative Commons Attribution International License (CC BY 4.0).

http://creativecommons.org/licenses/by/4.0/

Open Access

\begin{abstract}
As part of a better understanding of drying liquids within porous materials, measurements from 293 to $343 \mathrm{~K}$ of deionized water surface tension in air as a function of relative humidity are exposed. Experimental work was carried out using the pendant drop method coupled with image analysis within an adapted instrumented climatic chamber. Results show that surface tension linearly decreases when relative humidity increases. Although the effect of humidity is less compared to that of the temperature and even less compared to a surfactant impact, it must not be neglected and values have to be mentioned when dealing with water evaporation. Modifying surface tension also affects the pendant drop shape. The drying kinetics of the pendant drop volume and its outer shell are connected to this change of shape. Steam in the air can be assimilated to a wetting agent, hence a surfactant, and can be used in an environmental-friendly way to ease the drying stage. Indeed, the challenge is to limit the risk of cracking and damaging pieces during this crucial step in material processing.
\end{abstract}

\section{Keywords}

Pendant Drop, Surface Tension, Water, Relative Humidity, Temperature, Drying

\section{Introduction}

Surface tension is due to cohesive forces between liquid molecules. This phenomenon results from molecules at liquid-air interfaces which are missing some of their attractive interactions. In this particular configuration they are not in a stable energy state. The liquid adapts to this situation and surface tension dictates reshaping in order to minimize its area in contact with the surrounding atmosphere, leading to numerous known liquid behaviors [1] [2], for instance coffee 
stains [3], or drops coalescence [4] [5]. Another example is a porous material where water is used for shaping. In ceramic processing for instance, drying is a necessary step to remove the water before firing. Since water has a high surface tension, a badly controlled drying process can lead to final ceramic pieces with several defects such as cracks [6] [7]. Several parameters can be adjusted to reduce liquid-air surface tension, such as increasing temperature [8], or adding surfactants (wetting agents) [9]. Besides, there is another factor which impacts on the drying stage and thus on surface tension, namely relative humidity of the drying air. This last parameter is very often left aside. Indeed when drying a porous material piece, the set of temperature and relative humidity parameters is very often motivated by empirical observations and comes more under instinctive choices than theoretical explanations. Some publications in other scientific fields reported the effects of relative humidity on surface tension. For instance, the spreading dynamics of a drop of blood can be modified [10], as well as the surface tension of lung surfactant films [11]. From this point of view, where the effects of humidity are non-negligible, Erbil did underline and deplore the lack of relative humidity information in numerous recent papers when water drop evaporation was tackled as it can alter the evaporation rate [12]. However, PérezDíaz et al. recently provided some interesting behaviors about how partial pressure of water vapor acts on surface tension at the liquid water-air interface at $278,283,288$ and $293 \mathrm{~K}$ [13]. These data and those of the preceding examples were obtained at body temperature or at lower temperatures and could not be used in a drying study where, for instance, the temperature of $333 \mathrm{~K}$ is commonly used.

This paper provides results about how the relative humidity affects the liquid surface tension at water-air interface. Using the pendant drop technique, it describes surface tension vs. humidity from $293 \mathrm{~K}$ up to $343 \mathrm{~K}$. To our knowledge, this range of temperature has never been investigated and this study provides new useful information. Beyond the effect on surface tension, this study also aims to provide values for future studies. From the experimental data, a new phenomenological relation is proposed, which describes the evolution of the water-air surface tension in the 278 - $343 \mathrm{~K}$ temperature range and for a relative humidity between $20 \%$ and $100 \%$. The study also focuses on the water pendant drop drying kinetics. The pendant drop volume, as well as its outer shell during drying is presented at temperatures from $303 \mathrm{~K}$ up to $343 \mathrm{~K}$ and relative humidities from $35 \%$ up to $75 \%$.

\section{Materials and Methods}

\subsection{Climatic Chamber}

Surface tension measurements are made at a given temperature and relative humidity. An environmental chamber as shown in Figure 1 was used to set the temperature and the relative humidity of the drying air. The temperature is adjusted by armored heaters, associated with the chamber controller with an accuracy of $0.1 \mathrm{~K}$. Air humidity is provided by a boiler. Platinum psychrometric sensors 


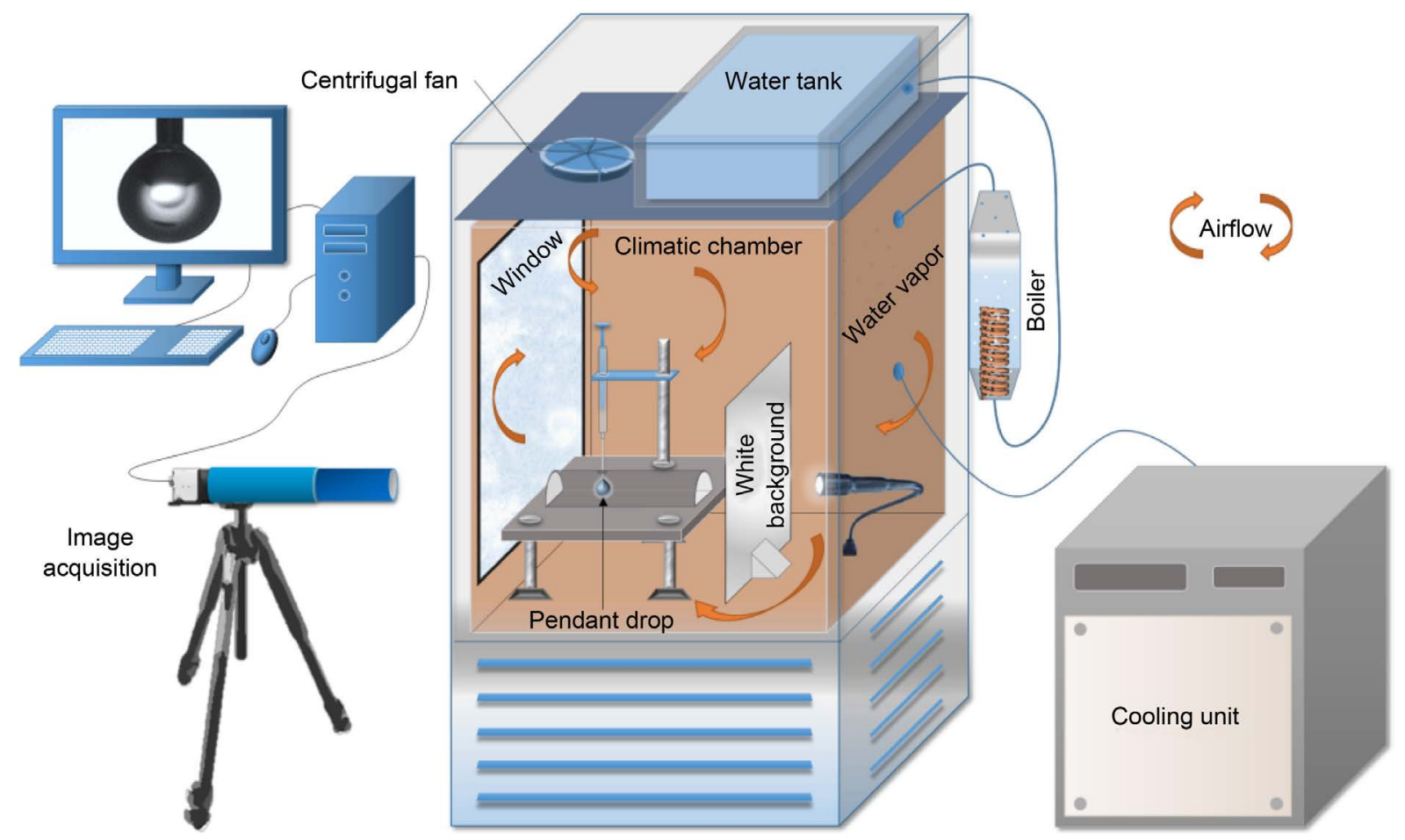

Figure 1. Experimental device for pendant drops measurements.

control the humidity of air with an accuracy of $0.1 \%$. If the relative humidity is higher than the set point, it is stabilized using a cooling unit linked to the chamber. The environmental chamber is computer controlled and data can be checked in real time. The pendant drop technique is used to determine the surface tension [14]. The climatic chamber has a zero diopter glass door to directly observe the pendant drop. As it was not possible to put a collimated light source into the chamber given the harsh conditions, a white background has been added to the system and a white led diffuses light to obtain a sufficient contrast to observe the pendant drops. The system of the climatic chamber is limited at low $(\sim 283 \mathrm{~K})$ and high $(\sim 353 \mathrm{~K})$ temperatures. Indeed, it is difficult to reach low relative humidity levels at low temperatures, and at high temperatures the cooling unit laboriously reaches the highest relative humidity levels. Therefore, results that are presented in this paper using this specific equipment are limited to temperatures between 293 and $343 \mathrm{~K}$ and relative humidities from $20 \%$ to $100 \%$. To avoid any temperature gradient, before creating a pendant drop, the liquid within the syringe is placed inside the climatic chamber so that it is at the same temperature as the target temperature.

\subsection{Pendant Drop}

In order to measure the surface tension of deionized water droplets, a drop was produced at the end of a capillary tube and elongated by gravity. There is then a balance between the hydrostatic pressure and the pressure determined by the Laplace equation. To make experiments with various temperatures and relative humidities, a $250 \mu \mathrm{L}$ Hamilton $^{\circledR}$ syringe was used to create pendant drops with 
an initial volume of $8.0 \mu \mathrm{L} \pm 0.1 \mu \mathrm{L}$. Deionized water (conductivity $<1 \mathrm{mS} \cdot \mathrm{m}^{-1}$ ) was used for drops and for the water tank providing humidity into the climatic chamber, thus the studied liquid and surrounding vapor have the same composition. Water density is of $1.000 \pm 0.005 \mathrm{~g} \cdot \mathrm{mL}^{-1}$ at $293 \mathrm{~K}$ and for the presented work it can decrease to $0.977 \pm 0.005 \mathrm{~g} \cdot \mathrm{mL}^{-1}$ at $343 \mathrm{~K}$. The pendant drop method is suitable in the case where the temperature or the relative humidity change [15]. Indeed, comparing to other methods, this is the most simple, accurate and reliable method for our equipment [16].

When realizing the surface tension measurements, the liquid is located within the syringe which is itself contained in the climatic chamber (Figure 2(a)). For each measurement at a given temperature and relative humidity, the system is left to equilibrate for at least 20 minutes. Then, the pendant drop is created and one image acquisition is realized immediately after. The evaporation process is considered to be negligible between the drop creation and the image acquisition. Indeed, the volume and the exchange surface of the pendant drop present almost no variations during the first three minutes of evaporation, as described later on Figure 11 and Figure 12. The literature reports a temperature discontinuity at the liquid-vapor interface when evaporating water, with the temperature always being higher on the vapor side. When considering a flat water/vapor interface instead of droplets at about $300 \mathrm{~K}$, the average difference ranges between 2.6 to $7.8 \mathrm{~K}$ at low relative humidity (i.e. <15\%) [17] [18]. In the case of evaporating droplets,

(a)

(b)

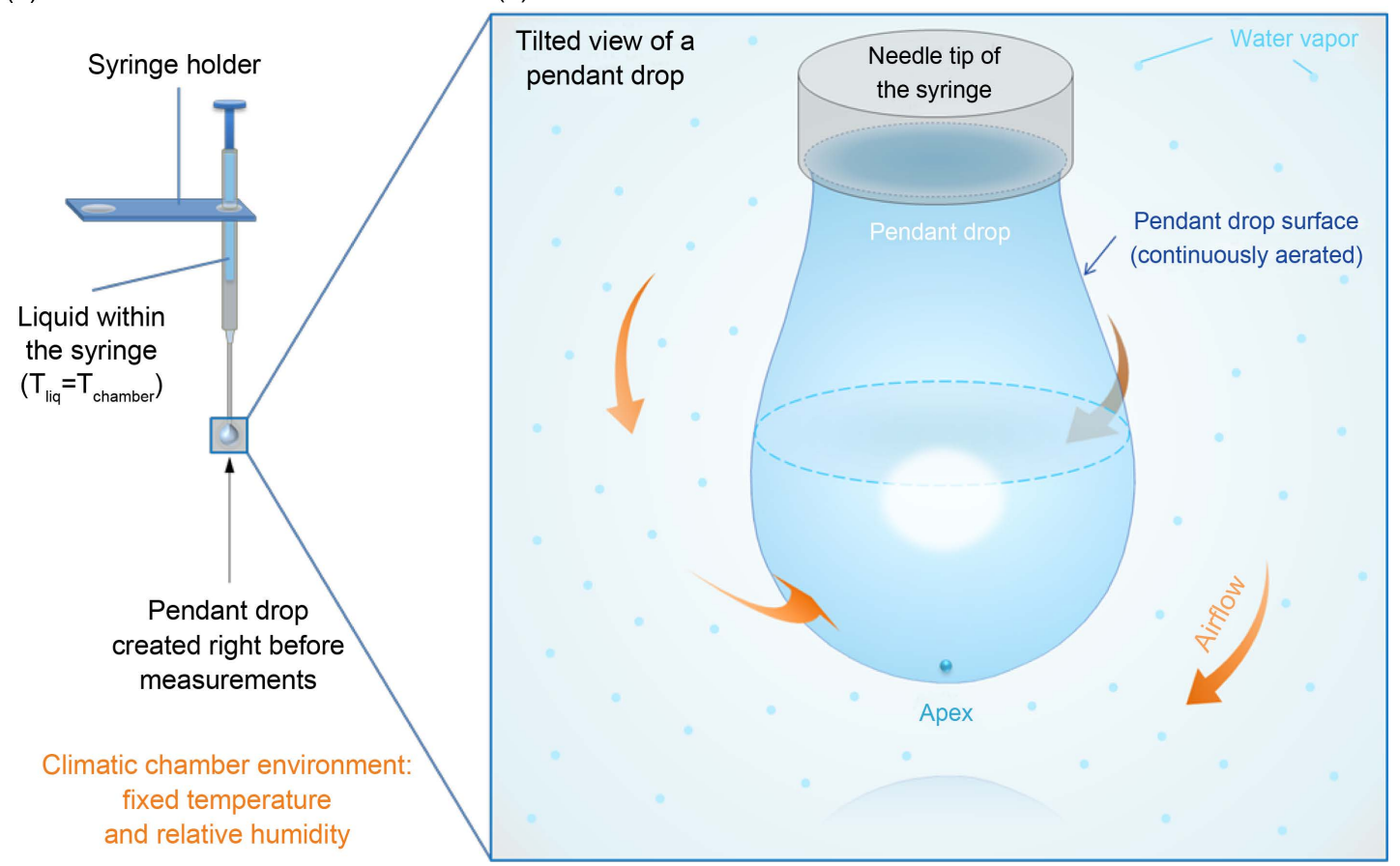

Figure 2. Conditions at the pendant drop surface: (a) deionized water is contained within the syringe before the creation of a pendant drop, so the environment temperature and the newly created drop are at the same temperature; (b) the air at the surface of the pendant drop is continuously renewed by the airflow within the chamber, so the relative humidity at the water-air interface is the same as the set relative humidity requested from the climatic chamber. 
literature reports an average difference below $1 \mathrm{~K}$ at high relative humidity (i.e. $>50 \%$ ) [19]. Therefore in the present work, there might exist a temperature offset in the presented values, but the observed trends are not impacted. As the liquid is contained within the syringe until the drop creation, the deionized water is at the same temperature as the target temperature required from the climatic chamber. Once the pendant drop is created, it is suspended in a controlled environment with a permanent airflow (Figure 2(b)). During all experiments at a given temperature and relative humidity, air is circulating within the climatic chamber and is permanently renewing the air around the pendant drop. Thus, the system is not static and the air at the surface of the drop is renewed continuously.

\subsection{Image Acquisition}

In order to obtain suitable images of the pendant drop at regular time intervals, a monochrome camera with charge-coupled device (CCD) was used, model UI-148SE-M from manufacturer IDS Imaging. The camera has a $5 \mathrm{M}$ pixel 1/2" sensor, a resolution of $2560 \times 1920$ pixels and a rate of 6 images $\cdot \mathrm{s}^{-1}$. It was coupled with an optical QIOPTIC which is made of two components, one model 35-08-06-000 and one model 35-00-03-000 which gives with a QIOPTIC 12.5:1 zoom an optical of $100 \mathrm{~mm} / 0.062$ 1/2".

\subsection{Surface Tension Data Processing}

The water liquid-air surface tension was obtained by analyzing the drop geometry using the Axisymmetric Drop Shape Analysis (ADSA) method [15] [20]. A representation of the ADSA routine is given on Figure 3. As water density changes with temperature, measurements are made using values from tables taken from the literature [21]. The analysis software created in the laboratory initially allows extracting the profile of the pendant drop (Figure 3(a)). In a first step a non-linear regression is realized on the profile which allows to access to a first value of surface tension (Figure $3(\mathrm{~b})$ ). This value can be compared afterwards using a second method where a simulated curve comes fitting the experimental

(a)

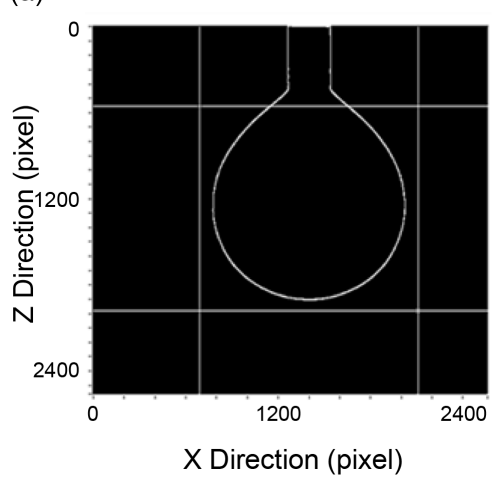

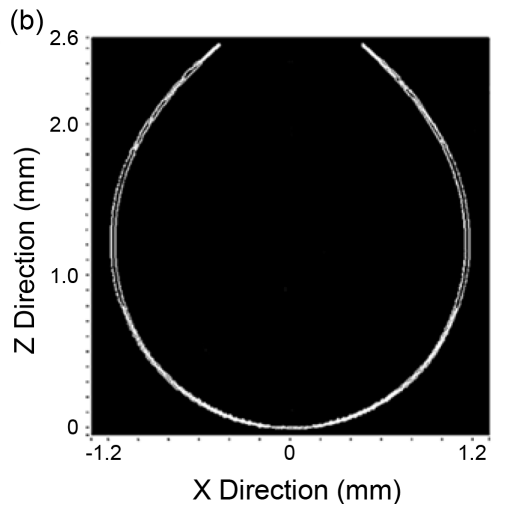

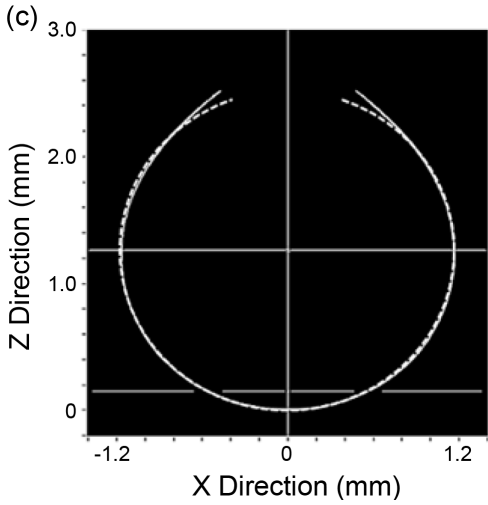

Figure 3. Example of ADSA routine on a deionized water drop: (a) digitalized image; (b) first method: non-linear regression; (c) fit realized with least squares method (the dotted line represents the simulated curve while processing). 
profile by adjusting precisely the position in the plan, the symmetry to the axis and the apex of the pendant drop. This last measurement uses least squares method and is carried out between two heights of drop (horizontal lines on Figure 3(c)) [22]. Moreover, the drop position, its rotation and the curvature at the apex are used as optimization parameters.

\subsection{Pendant Drop Volume and Area Evaluation}

The present study also proposes an evaluation of the volume and the area of the pendant drop during drying. Image analysis was used to realize these measurements, and consisted in a three steps procedure. The first step consisted in producing pendant drops with an initial volume of $8.0 \mu \mathrm{L} \pm 0.1 \mu \mathrm{L}$, which were observed during drying. The millimetric dimensions of the pendant drop are given in Figure 4(a). Images were taken each minute. In the second step, after the image acquisition, pictures were cropped in order to retain the liquid part and remove the syringe needle. As the pictures were given in grayscale, a threshold allowed to detect the edge of the pendant drop (Figure 4(b)). Finally in the third step, using the outer diameter of our Hamilton ${ }^{\oplus}$ syringe needle as a standard to convert pixels values in meters, namely $5.08 \times 10^{-4} \mathrm{~m}$, a program created in the laboratory then calculated the volume and the area of the pendant drop. More precisely, the pendant drop was divided into subsections and a circular revolution allowed the evaluation of all the sub volumes. The volume then resulted from their addition.

This method not only provided an accurate evaluation of the drop volume but also allowed to locate precisely the gravity center of each pendant drop sub volume, which minimized the effect of any shift due to additional micro-vibrations in the system associated to the convection within the climatic chamber. The pendant drop was then rebuilt in three dimensions (Figure 4(c)). To evaluate

Syringe outer diameter $(0.508 \mathrm{~mm})$

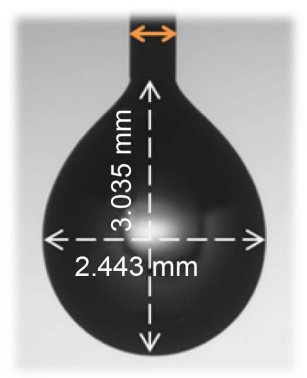

(a)

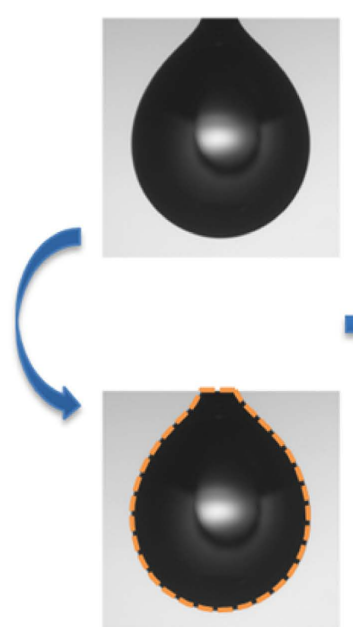

(b)

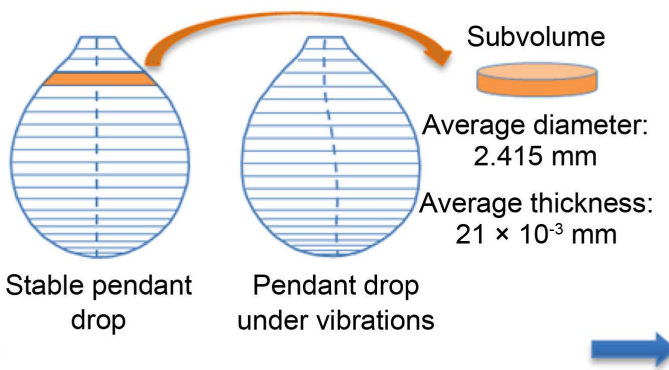

Three-dimensional reconstruction

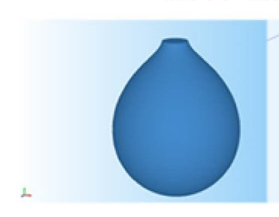

(c)

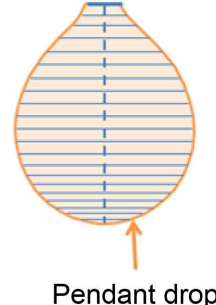

Pendant drop outer shell

Outer shell

(d)

Figure 4. Water pendant drop volume and area evaluation: (a) pendant drop of deionized water; (b) cropped image of the pendant drop followed by an edge detection (dashed line); (c) pendant drop volume evaluation (gravity center in dashed line) and three-dimensional reconstruction; (d) pendant drop outer shell evaluation. 
the exchange surface, only the outer shell was retained. Moreover, the surface in contact with the tip of the syringe needle was not considered in this evaluation (Figure 4(d)).

The pendant drop volume can influence the obtained surface tension values. Recently, Berry et al. introduced a new parameter, namely the Worthington number $W o$, in order to characterize the surface tension measurement precision [23]. First written as the ratio of the pendant drop volume to the maximum possible pendant drop volume, this dimensionless number is directly connected to the outer diameter of the syringe needle and pendant drops close to the critical detachment volume present the most accurate measurements. This number is written as presented in Equation (1):

$$
W o=\Delta \rho g V_{d} / \pi \gamma D_{n} \text {. }
$$

where $\Delta \rho=\rho_{d}-\rho$ is the density difference between $\rho_{d}$, the drop phase density and $\rho$ the continuous phase (air) density in $\mathrm{kg} \cdot \mathrm{m}^{-3} ; g$ is the standard acceleration due to gravity in $\mathrm{m} \cdot \mathrm{s}^{-2} ; V_{d}$ is the volume of the pendant drop in $\mathrm{m}^{3} ; \gamma$ is the water-air surface tension in $\mathrm{N} \cdot \mathrm{m}^{-1} ; D_{n}$ is the syringe needle outer diameter in $\mathrm{m}$.

When the volume is not sufficient, Wo values are much lower than 1. Good values of surface tension are obtained for Wo values greater than 0.6 . In the case of water considered at $293 \mathrm{~K}$ within an air saturated by vapor, the surface tension is equal to $72.75 \pm 0.36 \mathrm{mN} \cdot \mathrm{m}^{-1}$ [8]. A needle with an outer diameter of $5.08 \times 10^{-4} \mathrm{~m}$ gives a $W o$ value of about 0.7 , which is an acceptable value to evaluate surface tension from the different pictures.

The presented equipment and numerical tools enabled to carry out a set of measurements at different temperatures and relative humidities.

\section{Results and Discussion}

Results presented in Figure 5 show that surface tension at the water-air interface decreases as relative humidity increases. The obtained values are in good agreement and in the continuity of the work of Pérez-Díaz et al. at low temperatures represented by the first four lines at 278, 283, 288 and $293 \mathrm{~K}$ [13].

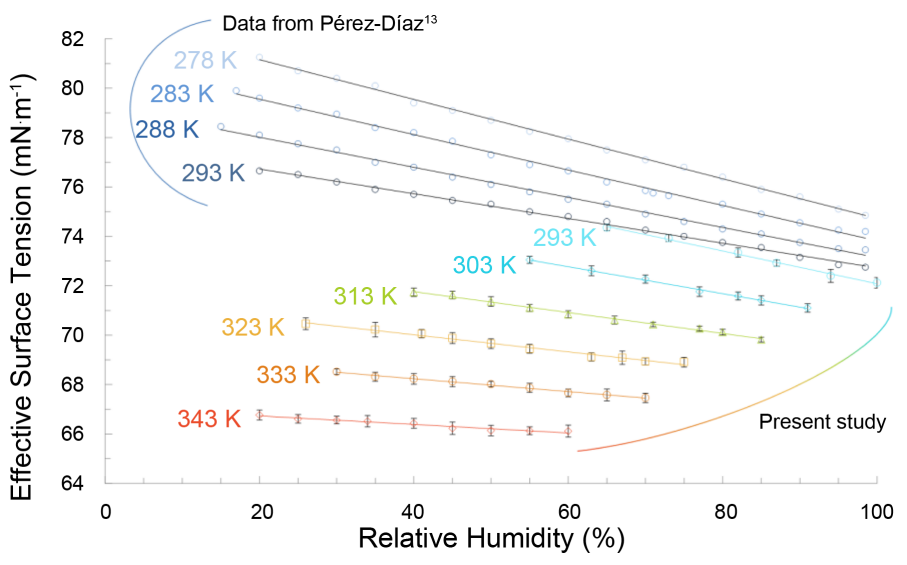

Figure 5. Evolution of surface tension as a function of the relative humidity. 
All other points presented in this chart are the results of the average of five measurements for each given temperature and relative humidity $H$. Vertical error bars correspond to the associated standard deviation. A linear dependency of $\gamma$ as a function of $H$ is observed. This effect is found to be less compared to the impact of increasing temperature. Besides, with increasing temperature, the relative humidity influences less and less surface tension, which can be observed on related slopes. In the following, we will examine the possible relations between the surface tension and the different parameters which can modify it, namely relative humidity, temperature and the addition of a surfactant.

\subsection{Relation between Relative Humidity and Surface Tension}

Pérez-Díaz et al. were the first to propose an interpretation of decreasing surface tension $\gamma_{l v}$ with increasing relative humidity [13]. $\gamma_{l v}$ is expressed in $\mathrm{N} \cdot \mathrm{m}^{-1}$ and represents the surface tension at liquid-vapor interface. Considering an infinite flat water surface or a surface element of a spherical water droplet in equilibrium with air at constant temperature and relative humidity (Figure 6), the forces at any point on the interface are described by Equation (2):

$$
\boldsymbol{F}_{\text {int }}+\boldsymbol{F}_{\text {ext }}=\mathbf{0} \text {. }
$$

where $\boldsymbol{F}_{\text {int }}$ (respectively $\boldsymbol{F}_{\text {ext }}$ ) corresponds to the forces applied perpendicularly to the surface by the liquid (respectively the air) just underneath (respectively above) the interface. Projecting according to the unitary normal $\boldsymbol{n}$ gives Equation (3):

$$
F_{\text {int }}=F_{\text {ext }} \text {. }
$$

where $F_{\text {int }}$ (respectively $F_{\text {ext }}$ ) corresponds to the norm of $\boldsymbol{F}_{\text {int }}$ (respectively $\left.\boldsymbol{F}_{\text {ext }}\right)$. However, it should be emphasized that in practice, when a droplet dries, there is a permanent flow of evaporating molecules that come from the liquid towards the exterior. If the liquid is under the form of a spherical droplet, $F_{\text {int }}$ from Equation (3) becomes Equation (4):

$$
F_{\text {int }}=4 \pi R_{\text {drop }}^{2} P_{\text {int }} .
$$

where $R_{\text {drop }}$ is the radius of the water droplet and $P_{\text {int }}$ is the internal pressure.

In this situation, $\boldsymbol{F}_{\text {ext }}$ has an additional term in order to take into account the tendency of the liquid to reduce its surface area (Equation (5)):

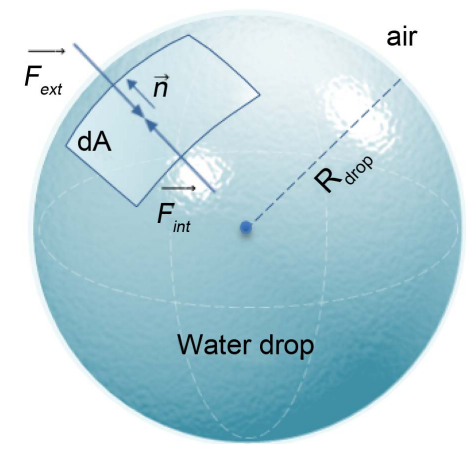

Figure 6. Representation of the exterior and interior forces at the drop surface. 


$$
F_{\text {ext }}=4 \pi R_{\text {drop }}^{2} P_{\text {ext }}+F_{\gamma} \text {. }
$$

where $P_{\text {ext }}$ is the external pressure outside the liquid and $F_{\gamma}$ is the norm of the force related to surface tension.

$F_{\gamma}$ is obtained by calculating the work necessary to diminish the radius of the spherical drop using Newton's mechanics (Equations (6) and (7)).

$$
\begin{gathered}
\mathrm{d} W_{\gamma}=F_{\gamma} \mathrm{d} R_{\mathrm{drop}} . \\
\mathrm{d} W_{\gamma}=\gamma \mathrm{d} A .
\end{gathered}
$$

where $A$ represents the area of the drop. Combining Equations (6) and (7), $F_{\gamma}$ can be written as follows (Equation (8)):

$$
F_{\gamma}=\gamma\left(\mathrm{d} A / \mathrm{d} R_{\mathrm{drop}}\right)=8 \gamma \pi R_{\mathrm{drop}} .
$$

Replacing all terms in Equation (3) using Equations (4), (5) and (8), it gives Equation (9):

$$
\gamma\left(2 / R_{\mathrm{drop}}\right)=P_{\mathrm{int}}-P_{\mathrm{ext}}=\Delta P .
$$

This is the standard equation of the equilibrium at liquid-air interface. It is important to underline that this equation is true only when interface is at equilibrium. Thus, considering Newton's third law about reciprocal actions, this flow can be directly assimilated to an overpressure in the air which aims to balance the ejection of water molecule during evaporation. Thus in the case of evaporating a liquid, a pressure term appears, $\delta P_{\text {evap }}$. Thus, Equation (9) becomes Equation (10):

$$
2 \gamma_{r} / R_{\text {drop }}=\lambda P+\delta P_{\text {evap }} \text {. }
$$

$\delta P_{\text {evap }}$ term was first proposed by Pérez-Díaz et al. [13]. It can also be written as the evaporation mass flow per unit area multiplied by the mean normal component of the speed of ejection of the molecules from the surface of the liquid (Equation (11)):

$$
\delta P_{\text {evap }}=f \cdot\left\langle v_{n}\right\rangle \text {. }
$$

where $f$ and $\left\langle v_{n}\right\rangle$ are respectively the mass flow per unit area in $\mathrm{kg} \cdot \mathrm{m}^{-2} \cdot \mathrm{s}^{-1}$ and the speed of ejection in $\mathrm{m} \cdot \mathrm{s}^{-1}$.

As there are more and more water molecules coming out of the liquid surface as the relative humidity of the drying air decreases at constant temperature, the mass flow can be rewritten as Equation (12):

$$
f=a(T)(1-H) \text {. }
$$

where $a(T)$ is a function of temperature $T$ (temperature of both the liquid and the environment) and $H$ is the relative humidity of the drying air. At this point, the assumption made by Pérez-Díaz et al. is to consider that the mean speed of ejection $\left\langle v_{n}\right\rangle$ depends only on the temperature which increases the kinetic agitation. Then, $\delta P_{\text {evap }}$ becomes Equation (13):

$$
\delta P_{\text {evap }}=a(T)\left\langle v_{n}\right\rangle(1-H) .
$$

Therefore, considering that $\gamma=\frac{\Delta P \times R_{\mathrm{drop}}}{2}$ given in Equation (9) is the initial 
value of surface tension when there is equilibrium (no evaporation) and combining Equation (13) with Equation (10), the effective surface tension $\gamma_{r}$ should be written as Equation (14):

$$
\gamma_{r}=\gamma+\left(a(T)\left\langle v_{n}\right\rangle R_{\text {drop }}\right)(1-H) / 2 .
$$

The drier the air is, the closer the droplet gets to the spherical shape given Figure 7(a). According to new expression of surface tension presented in Equation (14), when $H$ increases, $\gamma_{r}$ should decrease linearly. The water molecules surrounding the liquid are numerous, so there is a decrease of surface tension due to local overpressure in the air. An illustration of the mechanism is given Figure 7(b). Increasing relative humidity generates an outside overpressure compensated by the adsorption of water molecules at the liquid surface, thus reducing liquid-air surface tension. However, if Equation (14) allows a physical interpretation, it includes an unknown temperature related term $a(T)$ which makes it impossible to use in practice.

\subsection{Relation between Temperature and Surface Tension}

Publications underlined how temperature affects surface tension, when the liquid is pure water or binary mixtures of water and alcohols [8] [24]. When temperature rises, kinetic agitation and evaporation rate increase. Thereby, surface tension generally decreases for the molecular interactions become less important. In the case of water for example, the weak hydrogen bonds between two molecules are weakened when the temperature increases. In this situation bonds can break leading to a stronger effect than the decrease in surface tension due to relative humidity (Figure $7(\mathrm{c})$ ). Theoretical considerations given below are also detailed in literature [15] [25]. The system considered here is still a pure water drop surrounded by its vapor. In this system, there is a dependence on the unpredictable placement of the dividing interface between water and vapor because of the continuous exchanges between the two phases as illustrated Figure 8 .

According to Adamson [25], the expression of the surface energy $E^{S}$ as a function of temperature and surface tension is given by (Equation (15)):

$$
E^{S}=\gamma-T(\partial \gamma / \partial T)_{P} .
$$

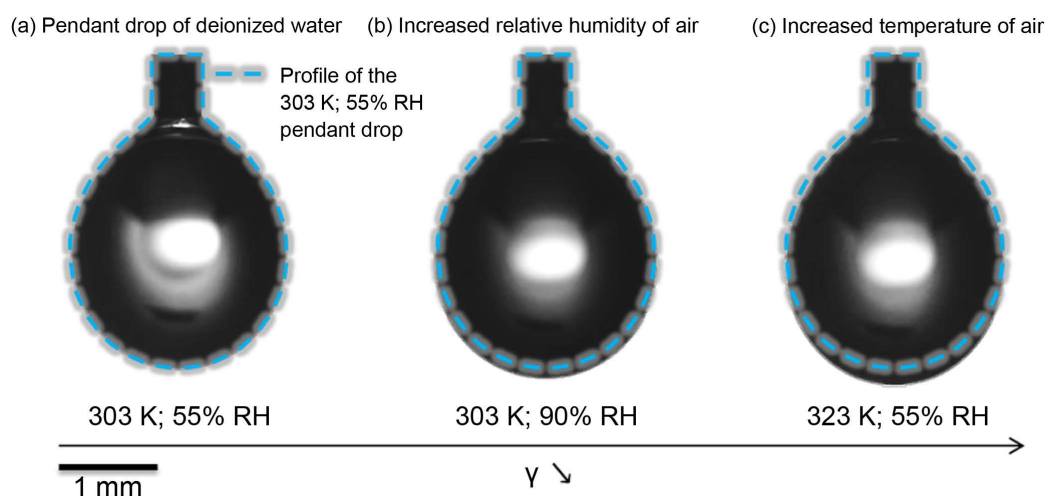

Figure 7. Example of two ways to decrease water liquid/air surface tension, from (a) maximum surface tension to (c) minimum surface tension (Bar: $1 \mathrm{~mm}$ ). 


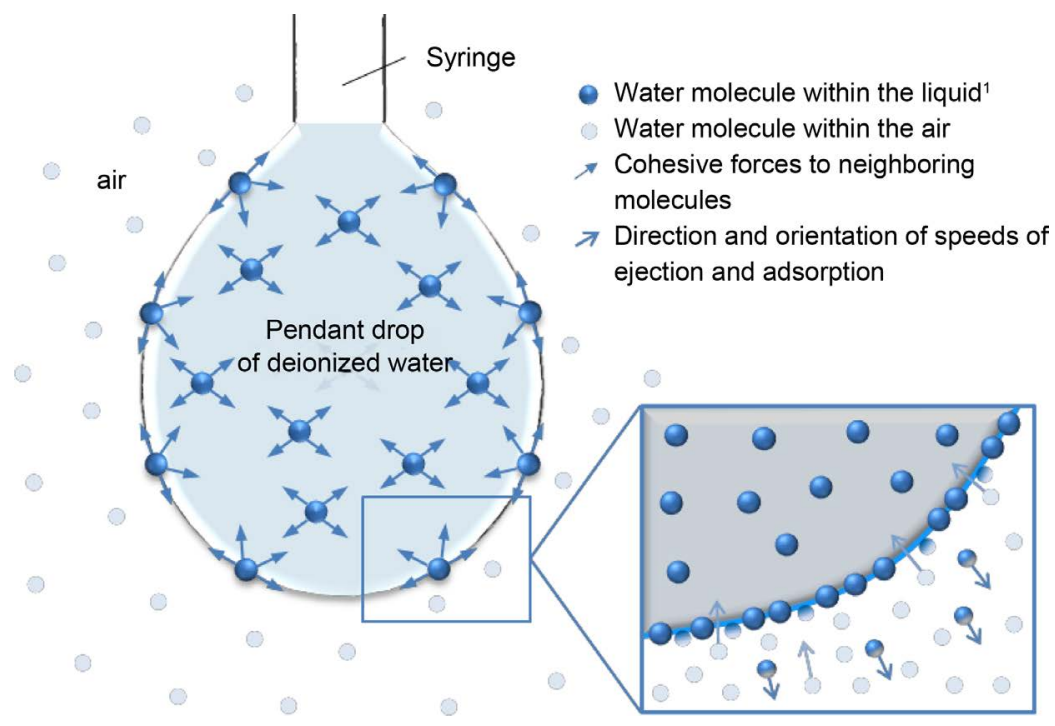

Figure 8. At the liquid-air interface, there is a continuous exchange between water molecules of the liquid phase and water molecules from the vapor phase.

It is observed that in the case of water, $\gamma$ decreases linearly with temperature [8]. This means that $E^{S}$ remains constant and equal to $\gamma$ when temperature increases until the temperature approaches the liquid's critical temperature $T_{C}$ where $E^{S}$ drops to zero. Empirical approaches used critical properties and molar volume to predict the surface tension of pure liquids, in the case where the air is saturated by the liquid's vapor. For instance, Eötvös derived an equation in 1886 by comparing the surfaces on the basis of the number of similarly shaped and symmetrically packed molecules per unit area [26] [27]. Then he proposed Equation (16):

$$
\gamma\left(V_{M}\right)^{2 / 3}=k\left(T_{C}-T\right) .
$$

where $V_{M}$ is the molar volume of the liquid in $\mathrm{m}^{3} \cdot \mathrm{mol}^{-1}, V_{M}=M_{W} / \rho_{L}, M_{W}$ and $\rho_{L}$ are the molar mass in $\mathrm{kg} \cdot \mathrm{mol}^{-1}$ and the liquid density $\mathrm{kg} \cdot \mathrm{m}^{-3}$ respectively. $k$ is the Eötvös constant with a typical value $2.1 \times 10^{-7} \mathrm{~J} \cdot \mathrm{K}^{-1} \cdot \mathrm{mol}^{-2 / 3}$ for non-associated liquids. In the case of water which is an associated liquid, $V_{M}=1.8 \times 10^{-5} \mathrm{~m}^{3} \cdot \mathrm{mol}^{-1}, T_{C}=647 \mathrm{~K}$ at a critical pressure of $22.3 \mathrm{MPa}$ and $k$ has a lower value which varies from $1.39 \times 10^{-7} \mathrm{~J} \cdot \mathrm{K}^{-1} \cdot \mathrm{mol}^{-2 / 3}$ at $273 \mathrm{~K}$ to $1.48 \times$ $10^{-7} \mathrm{~J} \cdot \mathrm{K}^{-1} \cdot \mathrm{mol}^{-2 / 3}$ at $343 \mathrm{~K}$.

However, it should be emphasized that this relation presented in Equation (16) and others resulting from it are empirical in nature. So far, nobody to our knowledge succeeded to propose a strict thermodynamically approach to predict the evolution of surface tension as a function of temperature [15]. Moreover, Equation (16) is only valid when air is saturated in vapor, and not for a specific relative humidity. Thus, Equations (14) and (16) cannot be used to calculate surface tension at any relative humidity or any temperature.

\subsection{Role of Relative Humidity as a Surfactant}

Another approach would be to consider the vapor phase as a wetting agent. 
Indeed, our experimental results show that steam can be assimilated to a surfactant as it lowers the surface tension. Without any additive, a water pendant drop is closer to a spherical shape (Figure 9(a)). Surfactants can be used in water to lower its surface tension [28]. They have a greater affinity for the surface than the volume medium. A surfactant can be described by the combination of a polar head group and a long carbon chain. At the surface, the molecules align so that the polar head is in contact with water and the carbon chain is oriented towards the air. When a surfactant is added at low concentration to a pure water droplet, the surface tension decreases as the amount of surfactant at the surface increases as illustrated in Figure 9(b).

This behavior is well described applying Langmuir adsorption isotherms to a water droplet mixed with a surfactant [29]. In this adsorption model, a continuous monolayer of adsorbed molecules surrounding a homogeneous surface is considered. In order to simplify, a neutral solute is considered, namely a surfactant. Adsorption isotherm expression at water/air interface is given by Langmuir equation and can be written as (Equation (17)):

$$
\Gamma=a C \Gamma^{S} /(1+a C) .
$$

where $\Gamma$ represents the amount of solute per unit area in $\mathrm{mol} \cdot \mathrm{m}^{-2}, \Gamma^{S}$ the adsorption at saturation in $\mathrm{mol} \cdot \mathrm{m}^{-2}\left(\Gamma=\Gamma^{S}\right.$ for a complete monolayer), $a$ is the Langmuir-Szyszkowski adsorption constant in $\mathrm{L} \cdot \mathrm{mol}^{-1}$ and $C$ the molar concentration of solute in $\mathrm{mol} \cdot \mathrm{L}^{-1}$.

Using the Gibbs-Duhem equation at constant temperature and composition allows writing Equation (18):

$$
\text { Ad } \gamma=-n_{i} \mathrm{~d} \mu_{i} .
$$

where $A$ is the area of the interface in $\mathrm{m}^{2}, \gamma$ is the liquid/air surface tension in $\mathrm{N} \cdot \mathrm{m}^{-1}, n_{i}$ in mol and $\mu_{i}$ in $\mathrm{J} \cdot \mathrm{mol}^{-1}$ are respectively the amount and the chemical

(a) Pendant drop of pure water

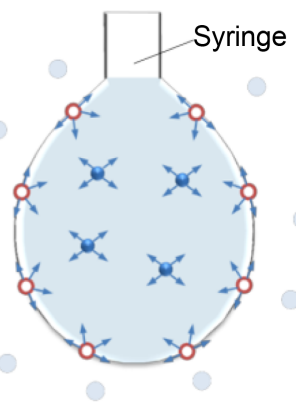

- Water molecule within the liquid

Water molecuke at the interface missing attractive interactions

Water molecule within the air

"Cohesive forces to neighboring molecules (b) Addition of an anionic surfactant

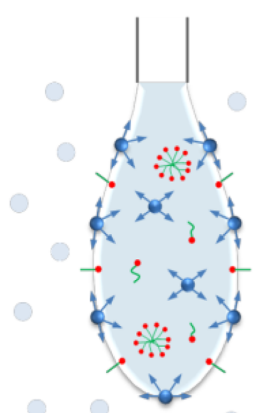

Hydrophobic tail of the molecule

- Hydrophilic head

: Agglomeration of molecules (micelle) after reaching the CMC

Figure 9. Effect of a surfactant on the surface tension of a water pendant drop: (a) without surfactant; (b) adding an anionic surfactant. 
potential of substance $i$ within the system. The chemical potential can be expressed as (Equation (19)):

$$
\mu_{i}=\mu^{0}+R T \ln C .
$$

where $\mu^{0}$ represents the chemical potential of substance $i$ in the standard state, $R$ is the ideal gas constant in $\mathrm{J} \cdot \mathrm{K}^{-1} \cdot \mathrm{mol}^{-1}, T$ the temperature in $K$ and $C$ is the absolute value of the molar concentration of the solute.

Combining Equations (18) and (19) gives another expression of interfacial surface tension (Equation (20)):

$$
\gamma=\gamma^{0}-\Gamma^{S} R T \ln (1+a C) .
$$

where $\gamma^{0}$ in $\mathrm{N} \cdot \mathrm{m}^{-1}$ is the interfacial tension when there is no solute mixed with the liquid. When no solute is added to the liquid, the surface tension value is $\gamma^{0}$. When there is a small amount of solute, $\gamma \approx \gamma^{0}-\Gamma^{S} R T a C$. For greater $C$ values, $\gamma \approx \gamma^{0}-\Gamma^{S} \ln (a C)$.

In other words, in the last case where the surface is saturated by surfactant molecules, the system then hits the critical micelle concentration (CMC). At this precise concentration, molecules start forming micelles (see Figure 9(b)). If the concentration exceeds CMC, then surface tension no longer changes. It is very common to use surfactants in ceramic processing as they act on surface tension, they also play an important part on the drying stage. Relations involving the surface concentration of surfactant to access surface tension of water/surfactant mixes are also proposed in the literature [30].

Nevertheless, the different presented expressions do not allow to directly use a relative humidity value.

\subsection{New Phenomenological Relation between Surface Tension, Temperature and Relative Humidity for Water}

Previous relations have shown it is not possible to predict the variations of surface tension when both the relative humidity and the temperature change. In order to show the surface tension dependence with temperature and relative humidity, two perspectives of a three dimensional representation are given in Figure 10 using a second order polynomial regression fitted on our experimental
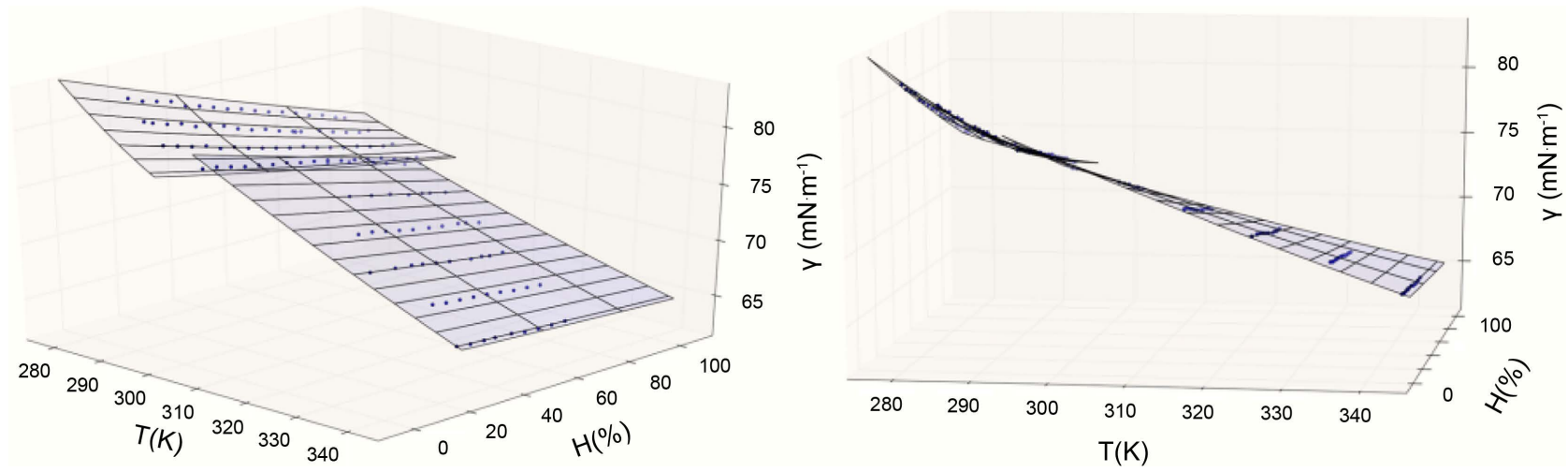

Figure 10. Representation of the $(\gamma, H, T)$ surfaces obtained by the second order polynomial regression applied on previous data. 
values. Data from Pérez-Díaz et al. study are also plotted [13], which gives an evolution of surface tension versus relative humidity from 278 to $343 \mathrm{~K}$. Both surfaces present two curvatures which show surface tension values cannot be given without precising both the temperature and relative humidity.

Regression coefficients obtained with the two sets of values are given on Table 1. Coefficients $a$ and $d$ related to relative humidity are lower than coefficients $b$ and $e$, related to temperature. Therefore temperature has a predominant effect. The equation contains a coupled term which underlines that the effects of temperature and relative humidity cannot be dissociated. The $d$ term is also negative which corresponds to the surfactant effect of relative humidity. Compared to other theoretical approaches presented in the literature [13] [25] [26], this phenomenological relation allows a numerical evaluation of the effective surface tension at a given temperature and relative humidity.

These two equations have been used in the case of saturated vapor ( $H$ is equal to 100) at different temperatures between 278 and $343 \mathrm{~K}$ and compared to experimental data from tables [8]. Results are presented in Figure 11. Experimental values obtained with ADSA method are close to data from tables between 278 and $333 \mathrm{~K}$. A deviation of about $1 \mathrm{mN} \cdot \mathrm{m}^{-1}$ is observed at $343 \mathrm{~K}$ which can be due to the lack of surface tension measurements at high relative humidities at this specific temperature. The practical interest in working in a humid environment is to decrease the surface tension of water and possibly avoid the use of organic surfactants when appropriate.

\subsection{Pendant Drop Volume and Area during Drying}

Measurements of $8 \mu \mathrm{L}$ pendant drops volume and surface exchange variations during drying were realized at temperatures from $303 \mathrm{~K}$ to $343 \mathrm{~K}$. Relative humidity was set to $55 \%$, which fits to a known value of a deionized water pendant drop surface tension for all tested temperatures. Pendant drops were hanging from a blunt needle tip which outer diameter was equal to $5.08 \times 10^{-4} \mathrm{~m}$. Results are presented in Figure 12. In order to illustrate the shape modification of the pendant drop during drying, three radii of curvature were considered (Figure 12(a)) referred as $r_{\text {left }}, r_{\text {right }}$ and $r_{\text {apex }}$ respectively taken at each side and at the apex of the pendant drop. The radii of curvature ratio, as defined on Figure 12(b), gets closer to 1 as the drying time increases. It means that the pendant drop get closer to a spherical shape. As expected, pendant drops dried faster as temperature gets higher (Figure 12(c)). The inset in Figure 12(c) shows that the drying time dramatically increases at the lowest temperatures. The dimensionless plot of the volume $v s$ the drying time presented in Figure 12(d)

Table 1. Coefficients of the second order polynomial regression realized on the $f(\gamma, H, T)$ plot. The equation is of the type $\gamma=a H^{2}+$ $b T^{2}+c T H+d H+e T+f$.

\begin{tabular}{cccccccc}
\hline Coefficients & $a$ & $b$ & $c$ & $d$ & $e$ & $f$ & \multicolumn{2}{c}{ Temperature range (K) Humidity range (\%) } \\
\hline Pérez-Díaz et al. study $[13] 6.937 \times 10^{-5} 4.005 \times 10^{-3} 2.045 \times 10^{-3}-6.580 \times 10^{-1}$ & -2.626 & $5.035 \times 10^{2}$ & {$[278 ; 293]$} & {$[15 ; 100]$} \\
\hline Present study & $1.759 \times 10^{-5} 3.938 \times 10^{-4} 9.701 \times 10^{-4}-3.503 \times 10^{-1}-4.792 \times 10^{-1} 1.852 \times 10^{2}$ & {$[293 ; 343]$} & {$[20 ; 100]$} \\
\hline
\end{tabular}




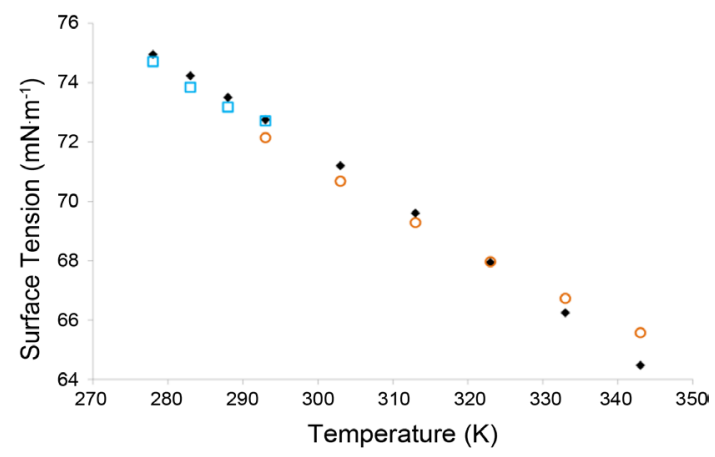

Figure 11. Comparison between surface tension values from tables and data obtained by polynomial regression at $\mathrm{H}=100 \%$ (values: $\downarrow$ data from tables [8]; $\square$ data from Pérez-Diaz et al. [13]; $\circ$ present study).
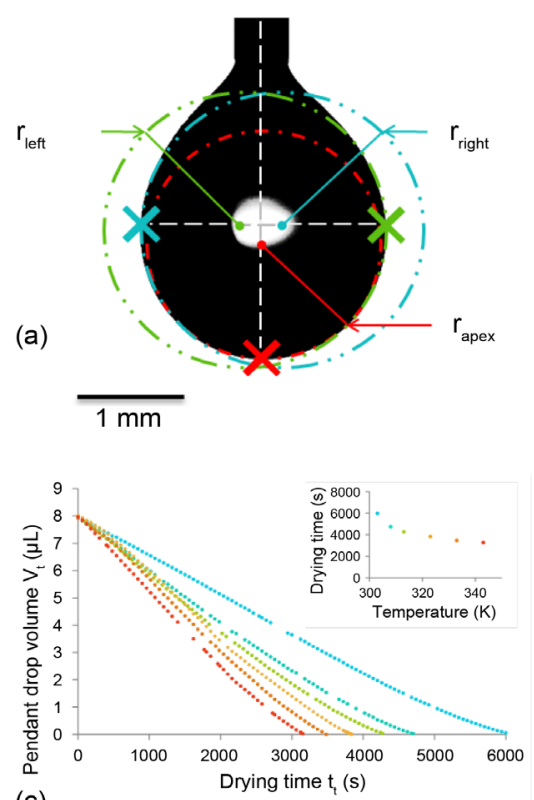

(c)

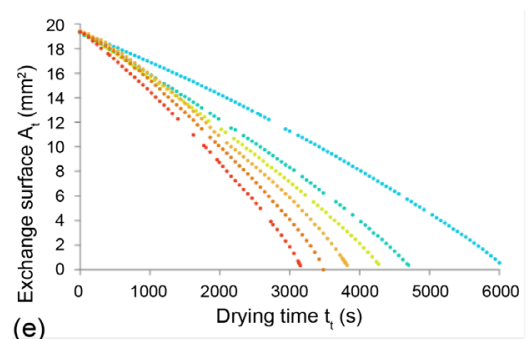

$303 \mathrm{~K}$ $308 \mathrm{~K}$

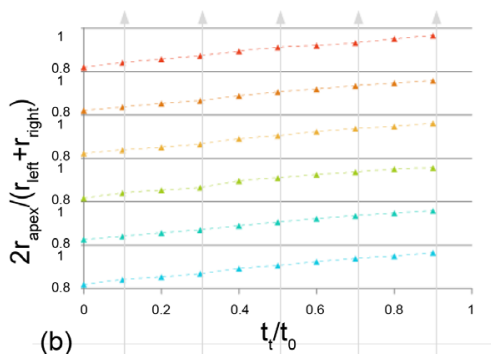

(b)

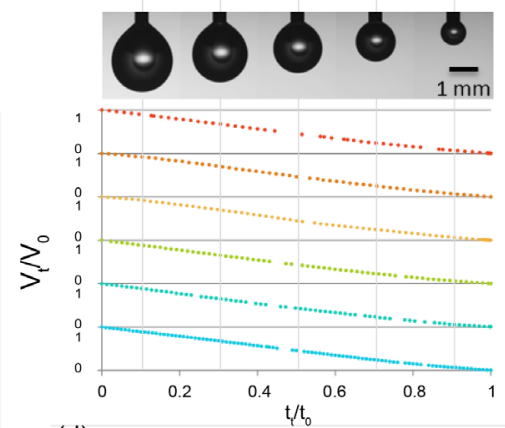

(d)

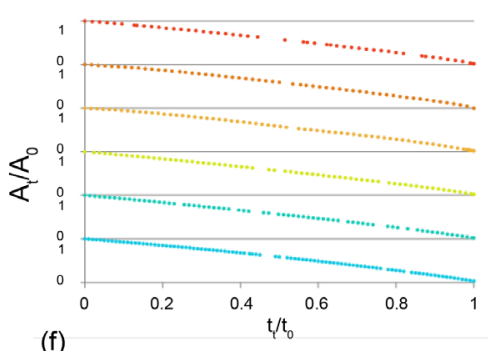

(f)

$323 \mathrm{~K}$

$343 \mathrm{~K}$

Figure 12. Evolution of $8 \mu \mathrm{L}$ pendant drops volume and surface exchange during drying at temperatures from $303 \mathrm{~K}$ to $343 \mathrm{~K}$. Relative humidity is set to $55 \%$ for all measurements: (a) illustration of the measured radii of curvature; (b) evolution of the radii of curvature ratio as a function of the dimensionless drying time (c) pendant drop volume as a function of the drying time (drying time as a function of the temperature in the inset); (d) dimensionless plot of the volume as a function of the drying time, illustration of the drop shape modification during the drying process; (e) pendant drop surface exchange as a function of the drying time; (f) dimensionless plot of the surface exchange as a function of the drying time. 
reveals that all curves follow the same trend. During the first minutes, the evaporation rate slightly decreases, as the drop was just created and is equilibrating with the environment. Then it increases and a second variation appears in the middle of the drying step, underlined by a change of curvature. The evaporation rate then decreases until the very last moments where the drop reaches the needle tip size and disappears in a few seconds. The change of curvature is attributed to the shape variations of the pendant drop revealed by the images in Figure 12(d). Indeed, it started as a bulb shape, where the drop volume is important and impacted by gravity, and progressively turned into a spherical shape where surface tension is predominant.

The curves of the outer shell of the pendant drops during drying do not present any change of curvature (Figure 12(e)). Initial surfaces are close to the surface of a perfect spherical drop of $8 \mu \mathrm{L}$, which is for information $19.34 \mathrm{~mm}^{2}$. These curves have a concave shape and as the volume (Figure 12(c)), the trend does not change as temperature gets higher (Figure 12(f)). Thus, temperature does not affect the behavior of pendant drops during drying but influences upon the kinetics. That is why two measurements were realized at a temperature of $323 \mathrm{~K}$, at $35 \%$ and $75 \%$ relative humidity.

Results are presented in Figure 13. The tendencies at the different relative humidities are similar. As expected the drying time dramatically increases with higher relative humidities. However, increasing the relative humidity up
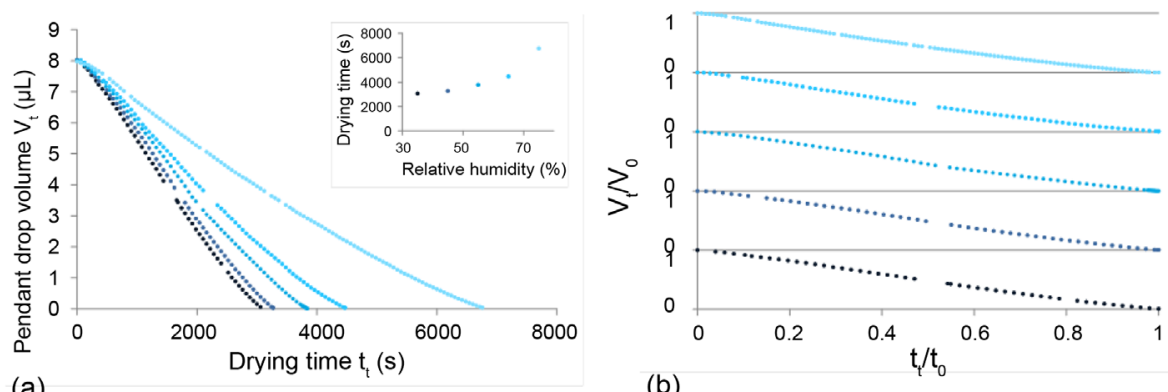

(a)

(b)
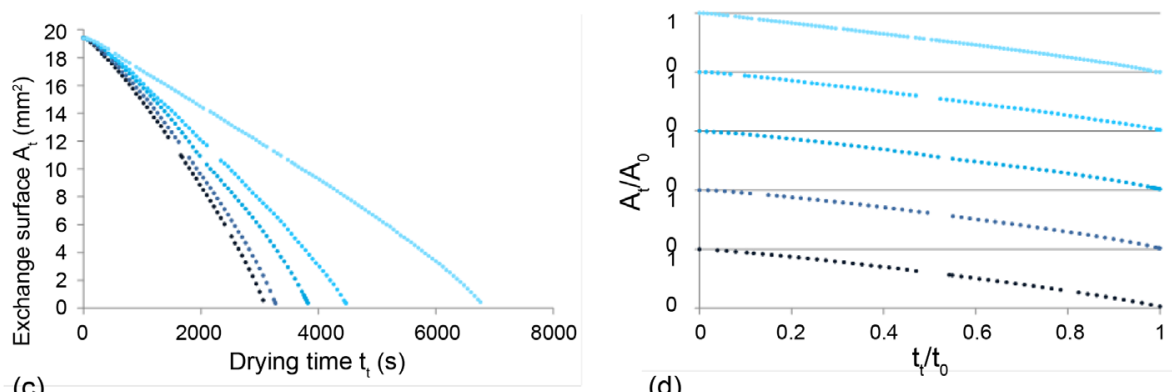

(d)

$323 \mathrm{~K} ; 35 \% \mathrm{RH}$

$323 \mathrm{~K} ; 45 \% \mathrm{RH}$

$323 \mathrm{~K} ; 55 \% \mathrm{RH}$

$323 \mathrm{~K} ; 65 \% \mathrm{RH}$

$323 \mathrm{~K} ; 75 \% \mathrm{RH}$

Figure 13. Evolution of $8 \mu \mathrm{L}$ pendant drops volume and surface exchange during drying at a set temperature of $323 \mathrm{~K}$. Relative humidity goes from $35 \%$ to $75 \%$ : (a) pendant drop volume as a function of the drying time (drying time as a function of the relative humidity in the inset); (b) dimensionless plot of the volume as a function of the drying time; (c) pendant drop surface exchange as a function of the drying time; (d) dimensionless plot of the surface exchange as a function of the drying time. 
to $75 \% \mathrm{RH}$ not only doubles the drying time compared to a relative humidity of $35 \%$ but also smoothes the curves. Indeed, the change of curvature observed on volume in Figure 12 is less visible at high relative humidities, as presented in Figure 13(a) and Figure 13(b). This is a direct consequence of the concentration gradient of water molecules within the vapor phase nearby the surface of the pendant drop, as illustrated previously in Figure 8. As relative humidity increases, the number of water molecules in the vapor phase then increases and limits the migration of the water molecules within the liquid phase toward the drying air. Moreover, the water surface tension decreases (Figure 5). Exchange surface evolution presented in Figure 13(c) and Figure 13(d) do not differ from those presented on Figure 12(e) and Figure 12(f).

\section{Conclusions}

Using pendant drop method within a humid environment, values of deionized water surface tension as a function of relative humidity between $20 \%$ and $100 \%$ have been calculated for temperatures from 293 to $343 \mathrm{~K}$. The pendant drop shape modifications are presented and phenomenological relations are proposed which allow an evaluation of surface tension in the $15 \%-100 \%$ humidity and 278 - $343 \mathrm{~K}$ temperature ranges. Indeed, reporting the experimental data on a three dimensional representation where surface tension is plotted as a function of temperature and relative humidity revealed the dependence of the surface tension to both parameters. New results are in continuity with those of the literature [13], realized at lower temperatures.

The pendant drop volume was calculated, not only as a post measurement check to ensure that the surface tension data were accurate but also to bring new experimental findings. The pendant drop behavior had only been studied in the case where the drop was small enough to form a spherical cap [31]. Here, measurements of the pendant drop volume and surface exchange vs the drying time at temperatures from $303 \mathrm{~K}$ to $343 \mathrm{~K}$ and relative humidities from $35 \%$ to $75 \%$ reveal how the change of droplet shape is linked to the different evolutions. Drying time decreases when temperature increases and relative humidity decreases. Focus must be maintained on the fact that steam can be assimilated to a surfactant and must be specified in the experimental procedure. In the case of drying a porous material, increasing humidity should not only slow the evaporation rate and increase the drying time but also lower the surface tension. Consequently, cracks formation could be reduced. Finally, we intend that the proposed study can provide new information for a predictive microscopic model close to real cases, such as a single water pendant drop or water within a porous material behavior subjected to different temperatures and relative humidities. The main objective is to give real meaning to realistic models whose parameters do not always have strict scientific values.

\section{Research Interest and Future Work}

Pendant drop tensiometry and image analysis allowed evaluating water drop 
behavior during drying, at different temperatures and relatives humidities. To our knowledge, such considerations were only discussed in the literature in the case where pendants drops were small enough to have the shape of a spherical cap [12] [31]. Indeed, volumes were less than $1 \mu \mathrm{L}$. When trying to provide an accurate representation of the fluid behavior at a mesoscopic scale, we noticed a lack of effective data that prevented us from proposing models as close as possible to reality at usual drying temperatures and relative humidities. Recently, modeling methods allowed realistic representation of fluids, whether in the case of modeling a liquid in contact with a substrate or just the liquid behavior [32] [33]. However, there is still no general approach to reproduce all the effects due to the interaction between the fluid and the air or the substrate. That is why these new insights could lead to more accurate models. The interest is also to have a better understanding of the fluid-substrate interface.

\section{Acknowledgements}

The authors thank Yoan Bertrand for contributing to the realization of the pendant drop test bench and data acquisition. The authors gratefully acknowledge the financial support provided by the Limousin region as part of the $\mathrm{PhD}$ of Etienne Portuguez.

\section{References}

[1] De Gennes, P.-G., Brochard-Wyart, F. and Quéré, D. (2004) Capillarity and Wetting Phenomena-Drops, Bubbles, Pearls, Waves. Springer-Verlag, New York. https://doi.org/10.1007/978-0-387-21656-0

[2] Clift, R., Grace, J.R. and Weber, M.E. (1978) Bubbles, Drops, and Particles. Academic Press, New York/London.

[3] Deegan, R.D., Bakajin, O., Dupont, T.F., Huber, G., Nagel, S.R. and Witten, T.A. (1997) Capillary Flow as the Cause of Ring Stains From Dried Liquid Drops. Nature, 389, 827-829. https://doi.org/10.1038/39827

[4] Eggers, J., Lister, J.R. and Stone, H.A. (1999) Coalescence of Liquid Drops. Journal of Fluid Mechanics, 401, 293-310. https://doi.org/10.1017/S002211209900662X

[5] Menchaca-Rocha, A., Martinez-Davalos, A. and Nunez, R. (2001) Coalescence of Liquid Drops by Surface Tension. Physical Review E, 63, Article ID: 046309. https://doi.org/10.1103/PhysRevE.63.046309

[6] Scherer, G.W. (1990) Stress and Fracture during Drying of Gels. Journal of NonCrystalline Solids, 121, 104-109. https://doi.org/10.1016/0022-3093(90)90113-Z

[7] Scherer, G.W. (1990) Theory of Drying. Journal of the American Ceramic Society, 73, 3-14. https://doi.org/10.1111/j.1151-2916.1990.tb05082.x

[8] Vargaftik, N.B., Volkov, B.N. and Voljak, L.D. (1983) International Tables of the Surface-Tension of Water. Journal of Physical Chemistry Reference Data, 12, 817 820. https://doi.org/10.1063/1.555688

[9] Adamson, A.W. and Gast. A.P. (1997) Physical Chemistry of Surfaces, 6th Edition, Wiley, New York. https://doi.org/10.1126/science.160.3824.179

[10] Bou-Zeid, W. and Brutin, D. (2014) Effect of Relative Humidity on the Spreading Dynamics of Sessile Drops of Blood. Colloids and Surfaces A, 456, 273-285. https://doi.org/10.1016/j.colsurfa.2014.05.004 
[11] Acosta, E.J., Gitiafroz, R., Zuo, Y.Y., Policova, Z., Cox, P.N., Hair, M.L. and Neumann, A.W. (2007) Effect of Humidity on Lung Surfactant Films Subjected to Dynamic Compression/Expansion Cycles. Respiratory Physiology \& Neurobiology, 155, 255-267. https://doi.org/10.1016/j.resp.2006.06.009

[12] Erbil, H.Y. (2012) Evaporation of Pure Liquid Sessile and Spherical Suspended Drops: A Review. Advances in Colloid and Interface Science, 170, 67-86.

https://doi.org/10.1016/j.cis.2011.12.006

[13] Pérez-Díaz, J.L., Álvarez-Valenzuela, M.A. and García-Prada, J.C. (2012) The Effect of the Partial Pressure of Water Vapor on the Surface Tension of the Liquid Water-Air Interface. Journal of Colloid and Interface Science, 381, 180-182. https://doi.org/10.1016/j.jcis.2012.05.034

[14] Stauffer, C.E. (1965) The Measurement of Surface Tension by the Pendant Drop Technique. The Journal of Physical Chemistry, 69, 1933-1938. https://doi.org/10.1021/j100890a024

[15] Erbil, H.Y. (2006) Surface Chemistry of Solid and Liquid Interfaces. Blackwell Publishing, Oxford.

[16] Drelich, J., Fang, C. and White, C. (2002) Measurement of Interfacial Tension in Fluid-Fluid Systems. In: Hubbard, A.T., Ed., Encyclopedia of Surface and Colloid Science, Marcel Dekker, Inc., New York, 3152-3166.

[17] Fang, G. and Ward, C.A. (1999) Temperature Measured Close to the Interface of an Evaporating Liquid. Physical Review E, 59, 417-428.

https://doi.org/10.1103/PhysRevE.59.417

[18] Ward, C.A. and Stanga, D. (2001) Interfacial Conditions during Evaporation or Condensation of Water. Physical Review E, 64, Article ID: 051509. https://doi.org/10.1103/PhysRevE.64.051509

[19] McGaughey, A.J.H. and Ward, C.A. (2002) Temperature Discontinuity at the Surface of an Evaporating Droplet. Journal of Applied Physics, 91, 6406-6415. https://doi.org/10.1063/1.1471363

[20] Rotenberg, Y., Boruvka, L. and Neumann, A. (1983) Determination of Surface Tension and Contact Angle from the Shapes of Axisymmetric Fluid Interfaces. Journal of Colloid and Interface Science, 93, 169-183. https://doi.org/10.1016/0021-9797(83)90396-X

[21] Kell, G.S. (1968) Density, Thermal Expansivity, and Compressibility of Liquid Water from 0 to $150^{\circ} \mathrm{C}$ : Correlations and Tables for Atmospheric Pressure and Saturation Reviewed and Expressed on 1968 Temperature Scale. Journal of Chemical \& Engineering Data, 20, 97-105. https://doi.org/10.1021/je60064a005

[22] Hartley, H.O. (1961) The Modified Gauss-Newton Method for the Fitting of Non-Linear Regression Functions by Least Squares. Technometrics, 3, 269-280. https://doi.org/10.1080/00401706.1961.10489945

[23] Berry, J.D., Neeson, M.J., Dagastine, R.R., Chan, D.Y.C. and Tabor, R.F. (2015) Measurement of Surface and Interfacial Tension Using Pendant Drop Tensiometry. Journal of Colloid and Interface Science, 454, 226-237. https://doi.org/10.1016/j.jcis.2015.05.012

[24] Vazquez, G., Alvarez, E. and Navaza, J.M. (1995) Surface Tension of Alcohol + Water from 20 to $50^{\circ} \mathrm{C}$. Journal of Chemical \& Engineering Data, 40, 611-614. https://doi.org/10.1021/je00019a016

[25] Adamson, A.W. (1986) A Textbook of Physical Chemistry, 3rd Edition, Academic Press, Inc., Orlando.

[26] Eötvös, R. (1886) Ueber den Zusammenhang der Oberflächenspannung der Flüssigkeiten mit ihrem Molecularvolumen. Annals of Physics, 263, 448-459. 
https://doi.org/10.1002/andp.18862630309

[27] Palit, S.R. (1956) Thermodynamic Interpretation of the Eötvös Constant. Nature, 177, 1180. https://doi.org/10.1038/1771180a0

[28] Rosen, M.J. (2012) Surfactants and Interfacial Phenomena. 4th Edition, Wiley, Hoboken. https://doi.org/10.1002/9781118228920

[29] Langmuir, I. (1918) The Adsorption of Gases on Plane Surfaces of Glass, Mica and Platinum. Journal of the American Chemical Society, 40, 1361-1403. https://doi.org/10.1021/ja02242a004

[30] Chang, C.-H. and Franses, E.I. (1995) Adsorption Dynamics of Surfactants at the Air/Water Interface: A Critical Review of Mathematical Models, Data, and Mechanisms. Colloids and Surfaces A Physicochemical and Engineering Aspects, 100, 1-45. https://doi.org/10.1016/0927-7757(94)03061-4

[31] Picknett, R. and Bexon, R. (1977) The Evaporation of Sessile or Pendant Drops in Still Air. Journal of Colloid and Interface Science, 61, 336-350. https://doi.org/10.1016/0021-9797(77)90396-4

[32] Tartakovsky, A. and Meakin, P. (2005) Modeling of Surface Tension and Contact Angles with Smoothed Particle Hydrodynamics. Physical Review E, 72, Article ID: 206301. https://doi.org/10.1103/PhysRevE.72.026301

[33] Akinci, N., Akinci, G. and Teschner, M. (2013) Versatile Surface Tension and Adhesion for SPH Fluids. ACM Transactions on Graphics, 32, Article No. 182. https://doi.org/10.1145/2508363.2508395

Submit or recommend next manuscript to SCIRP and we will provide best service for you:

Accepting pre-submission inquiries through Email, Facebook, LinkedIn, Twitter, etc. A wide selection of journals (inclusive of 9 subjects, more than 200 journals) Providing 24-hour high-quality service User-friendly online submission system Fair and swift peer-review system Efficient typesetting and proofreading procedure Display of the result of downloads and visits, as well as the number of cited articles Maximum dissemination of your research work

Submit your manuscript at: http://papersubmission.scirp.org/

Or contact ns@scirp.org 